\title{
Phase Identification Using Series of Selected Area Diffraction Patterns and Energy Dispersive Spectrometry within TEM
}

\author{
Kun-Lin Lin \\ National Nano Device Laboratories, Hsinchu, Taiwan \\ Email: kllin@narlabs.org.tw \\ Received 11 August 2014; revised 4 September 2014; accepted 8 October 2014 \\ Copyright (C) 2014 by author and Scientific Research Publishing Inc. \\ This work is licensed under the Creative Commons Attribution International License (CC BY). \\ http://creativecommons.org/licenses/by/4.0/

(c) (i) Open Access

\begin{abstract}
Transmission electron microscopy (TEM) is a very powerful technique for materials characterization, providing information relating to morphology, composition, and crystal structure. Selected area diffraction patterns (SADPs) are crystallographic data that can be obtained using a TEM instrument. Conventional identification through SADP/TEM is tricky and tedious, thereby increasing the difficulty of phase identification. To establish a procedure for phase identification of known and unknown phases, in this study we examined two samples: one, a known phase, was $\mathrm{Si}$ with $<100>$ alignment; the other, unknown, was the $\mathrm{Ti}_{x} \mathrm{O}_{y}$ phase at the 96.4Au-3Ni-0.6Ti interlayer/ yttria-stabilized zirconia (YSZ) interface of a steel/96.4Au-3Ni-0.6Ti interlayer/YSZ joint. The procedures for phase identification of the known and unknown phases are described herein using a series of SADPs and energy dispersive spectrometry within TEM that would be useful for general researchers.
\end{abstract}

\section{Keywords}

Phase Identification, Transmission Electron Microscopy, Selected Area Diffraction Pattern, Energy Dispersive Spectroscopy

\section{Introduction}

Analytical transmission electron microscopy (TEM) is a very useful technique for materials characterization because it can provide information relating to the morphology, composition, and crystal structure. Selected-area diffraction patterns (SADPs) are crystallographic data that can be obtained within a TEM instrument. SADP is employed to select the interest of the specimen to obtain its diffraction patterns in a screen that is projection of 
the reciprocal lattice of interest, appearing as sharp diffraction spots. In SADP, the brightest spot (the so-called transmitted spot) usually appears in the center of the diffraction pattern; the other spots are diffraction patterns. In general, one projected SADP can provide two-dimensional (2D) crystallographic information, such as two axes of the lattice parameters. To obtain complete crystallographic information, including lattice parameters of interest ( $a, b, c$, and relative angles), defects, and orientational relationships, it is necessary to tilt the sample to obtain a series of SADPs.

At present, most textbooks describe the fundamentals of SADP, such as its use to determine the angles and distances between diffraction patterns and the characterization of zone axes, superlattices, and pattern types [1][5]. For SADP through TEM, however, only a few textbooks [1] [2] introduce the indexing of SADP, but are not very clear in their description of the procedures for the indexing of SADPs. Although Fultz and Howe [3] introduced two methods- "start with zone axis" and "start with diffraction patterns"-for the indexing of SADPs, these approaches prefer crystals that are cubic systems, such as face-centered cubic (FCC) or body-centered cubic (BCC). In fact, the key point for the indexing of SADP is to establish a series of reference SADPs. Textbooks [1]-[5] make it easy to obtain reference SADPs of FCC, BCC, diamond, and hexagonally close-packed (HCP) structures for indexing with such crystals. For other crystal structures, including monoclinic, orthorhombic, and rhombohedral forms, it is very difficult to index and identify their SADPs. Thus, crystallographic software is very useful for evaluating and establishing the reference SADPs. Several crystallographic programs are currently available (e.g., JEMS, CaRIne Crystallography). Although it is commonly believed that reference SADPs for all crystals are easy to produce using such software, procedures remain to be established for matching experimental SADPs with the reference SADPs.

In this study, the purpose was not only to determine the indexing of an SADP but also to establish a procedure for phase identification using a series of SADPs and energy-dispersive spectrometry (EDS) through TEM. We have found that important information regarding phase identification can be recorded while operating the TEM, not only after taking several SADPs of interest. The identification of serial SADPs through TEM for unknown phases is tricky and tedious, thereby increasing the difficulty of phase identification. In this paper, we describe procedures for the phase identification of a known $\mathrm{Si}$ phase and an unknown $\mathrm{Ti}_{\mathrm{x}} \mathrm{O}_{\mathrm{y}}$ phase formed at the $96.4 \mathrm{Au}-$ 3Ni-0.6Ti interlayer/YSZ interface of the steel/96.4Au-3Ni-0.6Ti interlayer/YSZ joint.

\section{Experimental Procedure}

To establish procedures for the phase identification of known and unknown phases, two samples we selected to demonstrate: $\mathrm{Si}$ with $<100>$ alignment as the known phase and the $\mathrm{Ti}_{\mathrm{x}} \mathrm{O}_{\mathrm{y}}$ phase at the 96.4Au-3Ni-0.6Ti interlayer/YSZ interface of the steel/96.4Au-3Ni-0.6Ti interlayer/YSZ joint as the unknown phase. For the latter, the joining procedure consisted of 1 ) placing $96.4 \mathrm{Au}-3 \mathrm{Ni}-0.6 \mathrm{Ti}$ (numbers are weight percentages) as a metallic interlayer between yttria-stabilized zirconia (YSZ) and stainless-steel coupons under a normal load of approximately $0.3 \mathrm{~N}, 2$ ) heating in a vacuum of approximately $10^{-6}$ torr to a temperature approximately $20^{\circ} \mathrm{C}$ above the liquidus of the interlayer for $5 \mathrm{~min}$, and 3) slowly cooling to room temperature at a controlled rate. The unknown $\mathrm{Ti}_{\mathrm{x}} \mathrm{O}_{\mathrm{y}}$ phase formed at the $96.4 \mathrm{Au}-3 \mathrm{Ni}-0.6 \mathrm{Ti}$ interlayer/YSZ interface because the Ti atoms of the interlayer readily reacted with the $\mathrm{O}$ atoms from YSZ to form a titanium-oxide phase.

Cross-sectional TEM specimens of Si and the steel/96.4Au-3Ni-0.6Ti interlayer/YSZ joints were cut, ground, polished, and then ion-milled using standard procedures. The crystal structures of the $\mathrm{Si}$ and unknown $\mathrm{Ti}_{\mathrm{x}} \mathrm{O}_{\mathrm{y}}$ phases were both characterized by analyzing the SADPs accessed using TEM (model JEM 2010Fx, JEOL, Tokyo, Japan) and EDS (X-Max 80TLE, Oxford Instruments, London, UK). The Inorganic Crystal Structure Database (ICSD) and crystallographic software (CaRIne Crystallography 3.1) were used to identify the indexing of the SADPs of the Si and unknown $\mathrm{Ti}_{\mathrm{x}} \mathrm{O}_{\mathrm{y}}$ phases. To accurately calculate lattice parameters of interest, magnified TEM images were calibrated using a MAG*I*CAL reference standard sample from Norrox Scientific.

\section{Results and Discussion}

\subsection{Identification of Known Phase}

The first step in identification of a phase is to search for crystallographic information, such as lattice parameters, space groups, interplanar spacings of the $(h k l)$ plane, and the reference SADPs of a known phase from a textbook [1]-[6], from software, or from the internet. Then, through TEM observations, recording an SADP with a 
low-order zone axis is necessary to assist the pattern indexing of the known phase. The next priority work is to measure the angle between the diffracted spots and to calculate the $d$-spacing of the diffracted spots nearest the (000) transmitted spot. The formula for calculating the $d$-spacing of diffracted spots is as follows [1]-[3]:

$$
R d=\lambda L
$$

The distance, $R$, is measured from the diffracted spot to the (000) transmitted spot. Because the electron wavelength, $\lambda$, and the camera length, $L$, are constant, we can measure the distance $R$ and obtain the $d$-spacing of the diffracted planes. For example, if the value of $\lambda$ is $0.0251 \AA$ at $200 \mathrm{kV}$, the value of $L$ is $80 \mathrm{~cm}$, and $R$ is measured to be $0.74 \mathrm{~cm}$, then the $d$-spacing $(d)$ will be $2.7135 \AA$. At present, charge coupled device (CCD) cameras are generally used instead of conventional developed film to more readily measure the $d$-spacings of diffracted planes. If the imaging system of TEM were a conventional developed film, it would be necessary to memorize the camera length $(L)$ while recording the SADPs. If the TEM used a CCD camera to capture the SADPs, the $d$-spacing of the diffraction patterns should be measured on-site because the measurement software is not available to get for general user. In addition, accurate measurement of the $d$-spacing of diffracted planes using a CCD camera requires periodical calibration of the imaging system of the TEM instrument.

To begin indexing a known phase, one should measure the angles of diffracted planes and at least two independent, and not co-linear, diffraction spots. Figure 1(a) presents an example of a SADP of Si with a low-order zone axis, recorded using a CCD camera. Two diffraction spots, $d 1$ and $d 2$, nearest the (000) transmitted spot can be measured as having $d$-spacings of 2.70 and $3.04 \AA$, respectively. The angles of the diffracted spots in the Figure 1(a) were also measured. The second step of the indexing process involves searching a database for the $d$-spacings of the diffraction planes and the SADPs of Si [Figure 1(b) and Figure 1(c)]. If possible, all of the reference diffraction patterns should be listed with various zone axes and then matched to the experimental SADPs. After matching the references to the experimental SADP of Si in Figure 1(a), the $d$-spacings of $d 1$ and $d 2$ and the angles of the diffracted patterns were consistent with those of the reference SADP of Si along with a zone axis of [011]. Figure 1(d) displays the final indexing of the SADP of Si in Figure 1(a).

Using trial-and-error to match the experimental and reference SADPs is a necessary and significant step in phase identification. Thus, having access to a large number of reference SADPs and their related $d$-spacings and angles of diffracted spots will assist in the indexing the SADP; these references are, however, limited in textbooks [1] [2] [6], increasing the difficulty of indexing complex crystal structure to known phases. Much crystallographic software has been developed to aid the indexing of SADPs because the reference SADPs and interplanar spacings are easy to identify after inputting the lattice parameters of interest, which are readily obtained

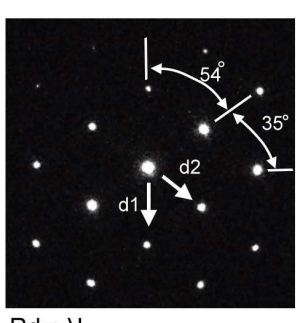

(a)

$$
\mathrm{Rd}=\lambda \mathrm{L}
$$

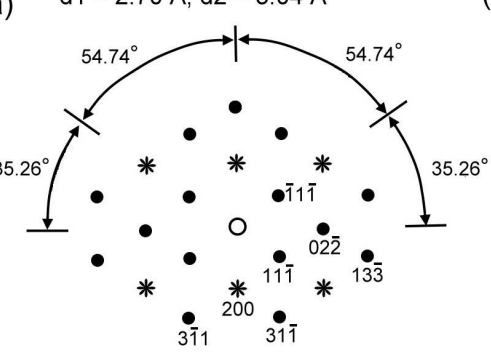

(c)

$$
Z=[011]
$$

\begin{tabular}{cc}
\hline Lattice parameter $(\AA)$ & $\mathrm{Si}$ \\
& 5.4282 \\
\hline$(h k l)$ & $\begin{array}{c}d \text { spacing } \\
(\AA)\end{array}$ \\
\hline 111 & 3.1340 \\
200 & 2.7142 \\
220 & 1.9194 \\
311 & 1.6365 \\
400 & 1.3571 \\
\hline
\end{tabular}

(b)

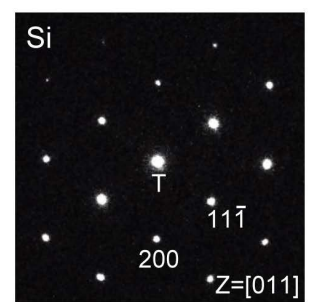

(d)

Figure 1. (a) SADP of Si; (b) (c) Interplanar spacings ( $d$-spacings) and SADP of Si [1]; (d) Final indexing of the SADP of Si. 
from the internet from crystallographic databases such as the crystallography open database or the inorganic crystal structure database (ICSD).

\subsection{Identification of Unknown Phase}

For identification of an unknown phase, the first step is using EDS to determine its composition, allowing database searching for related references. A series of SADPs from one grain of the unknown phase should then be recorded. Figure 2 displays the procedure for recording a series of SADPs with different zone axes for an unknown phase. To begin with, one grain of unknown phase should be tilted to a pole along with one zone axis, such as zone 1 in Figure 2; the next SADP should be tilted along with the Kikuchi line of $g 1$ in zone 1 or the $g 1$ spot should be kept strongly excited to the SADP with zones 2 and 3 . Next, one should return to zone 1 and tilt to zones 4 and 5 along with the Kikuchi line of $g 2$ of zone 1. Finally, a series of SADPs of unknown phase with five zone axes should be captured. In addition, the $(X, Y)$ coordinates of each zone axis should be recorded because the angle between the different zone axes can be calculated from these values. These $(X, Y)$ coordinates should be noted immediately on-site, because these values will not exist either on the developed film or on the digital image of the SADPs out of TEM observations. Moreover, it is necessary to label which SADPs belong to the low-order zone axes, revealing a large number of Kikuchi lines crisscrossed together in the Kikuchi pattern, as in zones 1, 3, and 5 of Figure 2. It is not necessary to draw detailed data of the serial SADPs, as in Figure 2, because it is time-consuming to perform on-site. It is, however, necessary to record the $(X, Y)$ coordinates along which the Kikuchi line is tilted and to at least label the low-order zone axis to help with indexing of the SADP.

Figure 3 presents a transmission electron micrograph (bright-field image, BFI) of the unknown $\mathrm{Ti}_{\mathrm{x}} \mathrm{O}_{\mathrm{y}}$ phase at the interface between the 96.4Au-3Ni-0.6Ti interlayer and YSZ of a steel/96.4Au-3Ni-0.6Ti interlayer/YSZ joint. Using EDS, the composition of the unknown $\mathrm{Ti}_{\mathrm{x}} \mathrm{O}_{\mathrm{y}}$ phase is composed of $42.2 \mathrm{at} \%$ of $\mathrm{Ti}, 53.7$ at $\%$ of $\mathrm{O}$, $1.0 \mathrm{at} \%$ of $\mathrm{Zr}$, and $0.1 \mathrm{at} \%$ of $\mathrm{Y}$. The next step involved recording a series of SADPs of the unknown $\mathrm{Ti}_{\mathrm{x}} \mathrm{O}_{\mathrm{y}}$ phase (Figure 4) and determining related information, as mentioned above. After TEM observation, we measured the $d$-spacing of the diffracted spots, the angle between the diffracted spots, and the tilting angle between the zone axes for the series of SADPs of the unknown $\mathrm{Ti}_{\mathrm{x}} \mathrm{O}_{\mathrm{y}}$ phase; Figure 4 also displays these calculated values. For instance, in zone axis (1), we measured the two diffracted spots nearest the (000) transmitted spot, labeled as " $T$ ", and the angles between diffracted spots, with values of $4.28 \AA, 2.73 \AA, 59.0^{\circ}$, and $33.4^{\circ}$, respectively. The low-order zone axis was labeled in the SADP with zone axes (2), (4), and (8) of Figure 4, according to the many Kikuchi lines crossing together in the Kikuchi patterns on TEM observations. Figure 4 also displays the calculated tilting angles between the two zone axes; for example, $22.1^{\circ}$ for zone axes (1) and (2), $16.6^{\circ}$ for zone axes (2) and (3), and $18.3^{\circ}$ for zone axes (2) and (7). Figure 5 presents the method for calculating the tilt-

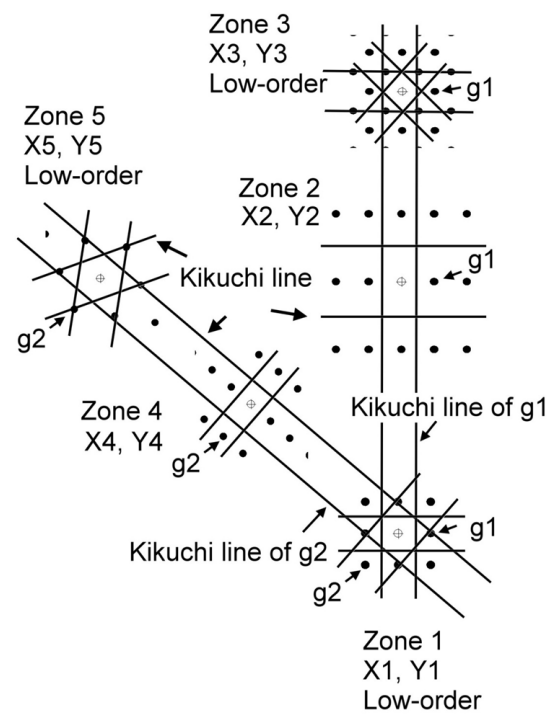

Figure 2. Procedure for taking a series of SADPs with different zone axes for an unknown phase. 


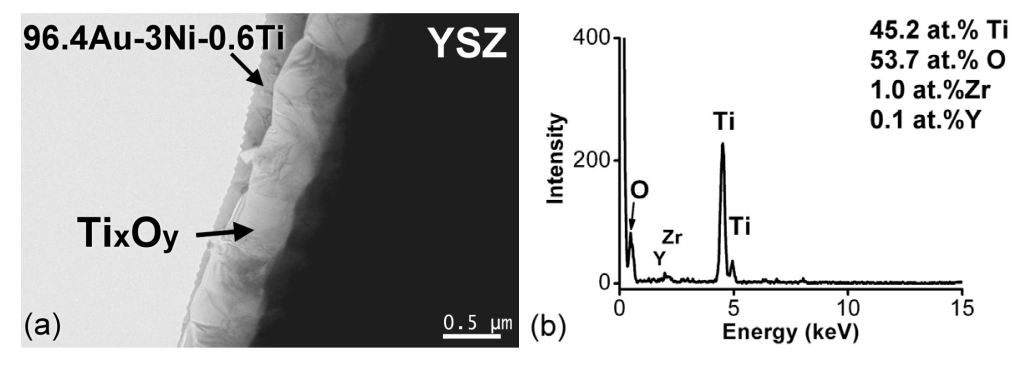

Figure 3. (a) Transmission electron micrograph (BFI) of the unknown $\mathrm{Ti}_{\mathrm{x}} \mathrm{O}_{\mathrm{v}}$ phase at the $96.4 \mathrm{Au}-3 \mathrm{Ni}-0.6 \mathrm{Ti}$ interlayer/YSZ interface of a steel/96.4Au-3Ni$0.6 \mathrm{Ti}$ interlayer/YSZ joint; (b) EDS spectrum of the unknown $\mathrm{Ti}_{\mathrm{x}} \mathrm{O}_{\mathrm{y}}$ phase.

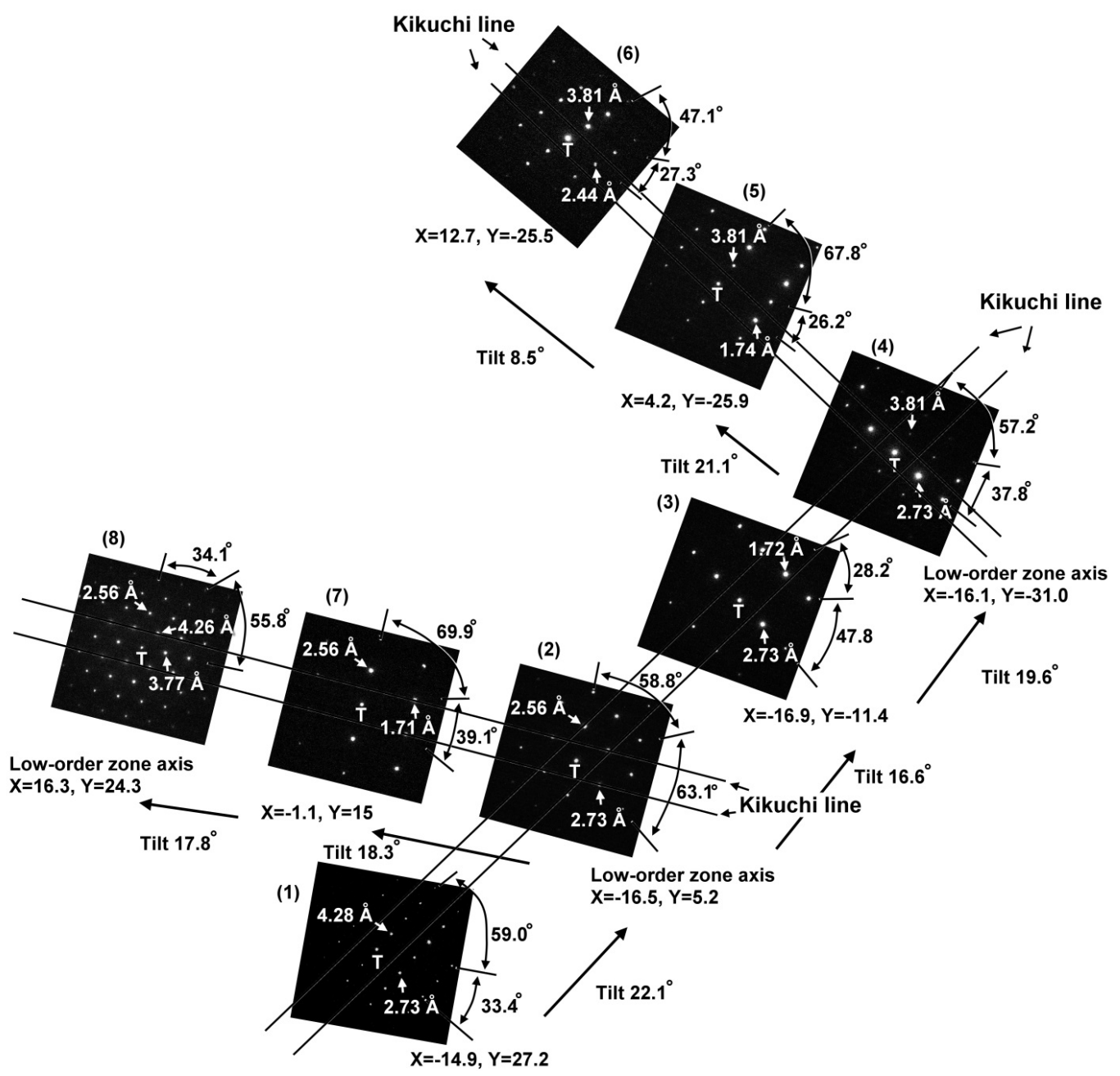

Figure 4. Series of SADPs of the unknown $\mathrm{Ti}_{\mathrm{x}} \mathrm{O}_{\mathrm{y}}$ phase.

ing angle between two zones. The angle between zones 1 and 2 is the distance between the two zones in the 2D $(X, Y)$ axis, projected by three-dimensional (3D) coordinates. Using the Pythagorean theorem, the distance, or angle, of zones 1 and 2 can be calculated using the equation

$$
\text { Angle }=\sqrt{(X 1-X 2)^{2}+(Y 1-Y 2)^{2}} .
$$

here, $(X 1, Y 1)$ and $(X 2, Y 2)$ are the $(X, Y)$ coordinates of the zone axes 1 and 2, respectively. Again, it is important to record the $(X, Y)$ coordinates of each zone axis on TEM observations, otherwise the information relating 

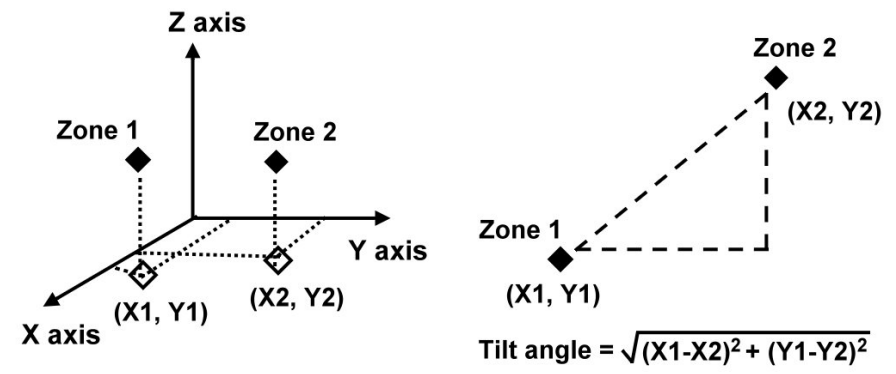

Figure 5. Calculation of the tilting angle between two zone axes.

to the tilting angle will be lost. Because the holder is side entry stage, the $X$ and $Y$ tilts are really not equivalent to exactly determine the specimen tilt from goniometer readings. Thus, the actual tilt angle is not obtained by the equation of Figure 5 but it is acceptable to get the tilt angle using the equation because the deviation just about 1 - 2 degrees evidenced by the tilt angle calculation of a known phase, Si.

At this stage, the composition and a series of SADPs of the unknown $\mathrm{Ti}_{\mathrm{x}} \mathrm{O}_{\mathrm{y}}$ phase have been obtained [Figure 3(b) and Figure 4]. The next step is to establish the crystal structure and lattice parameters of the possible $\mathrm{Ti}_{\mathrm{x}} \mathrm{O}_{\mathrm{y}}$ phase, using a database such as the crystallography open database or the ICSD, as listed in Table 1. Next, the trial-and-error method is used to match the experimental SADPs in Figure 4 with the simulated or reference SADPs of the possible $\mathrm{Ti}_{\mathrm{x}} \mathrm{O}_{\mathrm{y}}$ phase. It is, however, difficult to search a series of reference SADPs of all the $\mathrm{Ti}_{\mathrm{x}} \mathrm{O}_{\mathrm{y}}$ phases in Table 1 from textbooks or reference articles. In contrast, a series of simulated SADPs of unknown phase is easy to produce using crystallographic software once we know some crystallographic information, such as the crystal structure and lattice parameters. Thus, many SADPs with different zone axes can be simulated, but those with low-order zone axes are preferred for initial simulation to quickly match with the experimental SADPs of low-order zone axes. The low-order zone axes probably belong to the $\langle 100\rangle,\langle 110\rangle$, $\langle 111\rangle$, $<120>$, and $<112>$ families. Figure 6 presents a series of simulated $\mathrm{SADPs} \mathrm{TiO}_{2}$ along with the [10 $\left.\overline{1}\right]$, [100], and [2 11 ] zone axes. The software should immediately record the $d$-spacing of two diffracted spots nearest the (000) transmitted spot, the angle between the diffraction spots, and the angle between the two zone axes. Next, the simulated SADPs of $\mathrm{TiO}_{2}$ along the [10 $\left.\overline{1}\right]$, [100], and [2 $\left.\overline{1} 1\right]$ zone axes in Figure 6 should be compared with the experimental SADPs with zones 2, 4, and 8 (the low-order zone axes) of Figure 4, whether any one set of SADPs is matched or not. The simulated SADP along with the [100] zone axis in Figure 6 appears to match the SADP along with zone 2 of Figure 4, because their $d$-spacings, angles, and pattern shapes are similar. For instance, in Figure 6 the $d$-spacings of the two diffracted spots in the simulated SADPs with the [100] zone axis are 2.293 and $2.483 \AA$; for the experimental SADPs with zone axis (2) of Figure 4, the $d$-spacings of the two spots were 2.56 and $2.73 \AA$. In terms of the angles of the diffracted spots, from Figure 6 we measured values of $57.21^{\circ}$ and $65.57^{\circ}$ for the simulated SADPs with the zone axis of [100]; in Figure 4, the values are $58.8^{\circ}$ and $63.1^{\circ}$ for the experimental SADPs with zone axis 2. Although one set of SADPs might be matched (the SADP with [100] in Figure 6 and that with zone 2 in Figure 4), we must still confirm the matching of the whole set of simulated and experimental SADPs. The angle of the zone axis of $[100]$ to the $[10 \overline{1}]$ zone axis along with the $(0 \overline{2} 0)$ plane in Figure 6 is $32.79^{\circ}$; this value is consistent with that of the experimental SADP with the zone axes (2) and (8) in Figure $4\left(18.3^{\circ}+17.8^{\circ}=36.1^{\circ}\right)$. Similarly, the angle between the simulated SADP with the zone axes of [100] and [2 $\overline{1} 1]$ in Figure 6 is $30.74^{\circ}$, similar to the angle of the experimental SADP with zones (2) and (4) $\left(16.6^{\circ}+19.6^{\circ}=36.2^{\circ}\right)$. Unfortunately, the shape of the simulated SADPs of zone axes of $[10 \overline{1}]$ and [211] of Figure 6 does not match that of the experimental SADP of the zone axes (8) and (4) in Figure 4. Thus, the $d$-spacings of their diffracted spots and the angles between the two spots are different. Consequently, this comparison of the simulated and experimental SADPs suggests that the unknown $\mathrm{Ti}_{x} \mathrm{O}_{y}$ phase is not $\mathrm{TiO}_{2}$.

Figure 7 displays another simulated SADP of TiO along with [210], [110], and [121] zone axes. Following the procedure described above, the simulated SADPs of TiO along with the [210], [110], and [121] zone axes in Figure 7 do not match with the experimental SADPs of zones (8), (2), and (4) in Figure 4, including the $d$ spacings of the diffracted spots, the angles between the two spots, and the tilting angles between the two zone axes. The next step was to use the trial-and-error method to compare the simulated SADPs of all possible Ti-O phases, based on Table 1, with the experimental SADPs of the unknown $\mathrm{Ti}_{\mathrm{x}} \mathrm{O}_{\mathrm{y}}$ phase. 
Table 1. Crystal structure and lattice parameters of possible $\mathrm{Ti}_{\mathrm{x}} \mathrm{O}_{\mathrm{y}}$ phases.

\begin{tabular}{ccc}
\hline Ti-O phase ICSD \# & Crystal structure space group & Lattice parameters \\
\hline $\mathrm{TiO}_{2}$ (rutile) 202241 & Tetragonal $\mathrm{P} 4 / 2 / \mathrm{mnm}(136)$ & $a=b=4.586 \AA ; c=2.954 \AA ; \alpha=\beta=\gamma=90^{\circ}$ \\
$\mathrm{TiO}_{2}$ (anatase) 024276 & Tetragonal I4 $/$ amd (141) & $a=b=3.776 \AA ; c=9.486 \AA ; \alpha=\beta=\gamma=90^{\circ}$ \\
$\mathrm{TiO}_{2}$ (brookite) 036411 & Orthorhombic Pbca (61) & $a=9.211 \AA ; b=5.472 \AA ; c=5.171 \AA ; \alpha=\beta=\gamma=90^{\circ}$ \\
$\mathrm{Ti}_{3} \mathrm{O}_{5} 075194$ & Monoclinic C2/m (12) & $a=9.756 \AA ; b=3.800 \AA ; c=9.438 \AA ; \alpha=\gamma=90^{\circ} ; \beta=91.547^{\circ}$ \\
$\mathrm{TiO}^{\circ} 048130$ & Cubic Fm3m (225) & $a=b=c=4.185 \AA ; \alpha=\beta=\gamma=90^{\circ}$ \\
$\mathrm{Ti}_{4} \mathrm{O}_{7} 019017$ & Triclinic A $\overline{1}$ & $a=5.590 \AA ; b=7.128 \AA ; c=12.48 \AA ; \alpha=95.03^{\circ} ; \beta=95.34^{\circ} ; \gamma=108.89^{\circ}$ \\
$\mathrm{Ti}_{2} \mathrm{O}_{3} 9658$ & Rhombohedral R $\overline{3}$ c (167) & $a=b=5.126 \AA ; c=13.914 \AA ; \alpha=\beta=90^{\circ} ; \gamma=120^{\circ}$
\end{tabular}

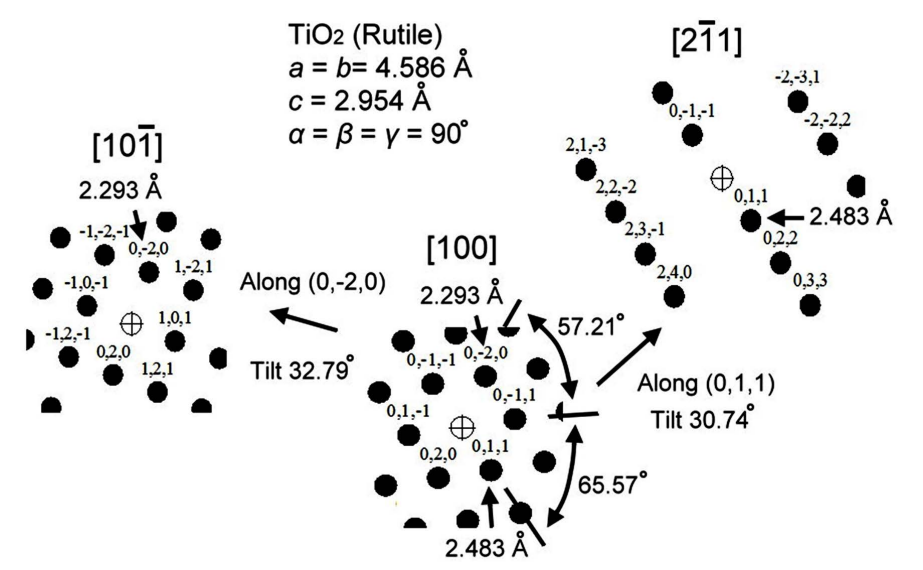

Figure 6. Simulated SADPs of $\mathrm{TiO}_{2}$ along the [10 $\left.\overline{1}\right]$, [100], and [2 $\left.\overline{1} 1\right]$ zone axes.

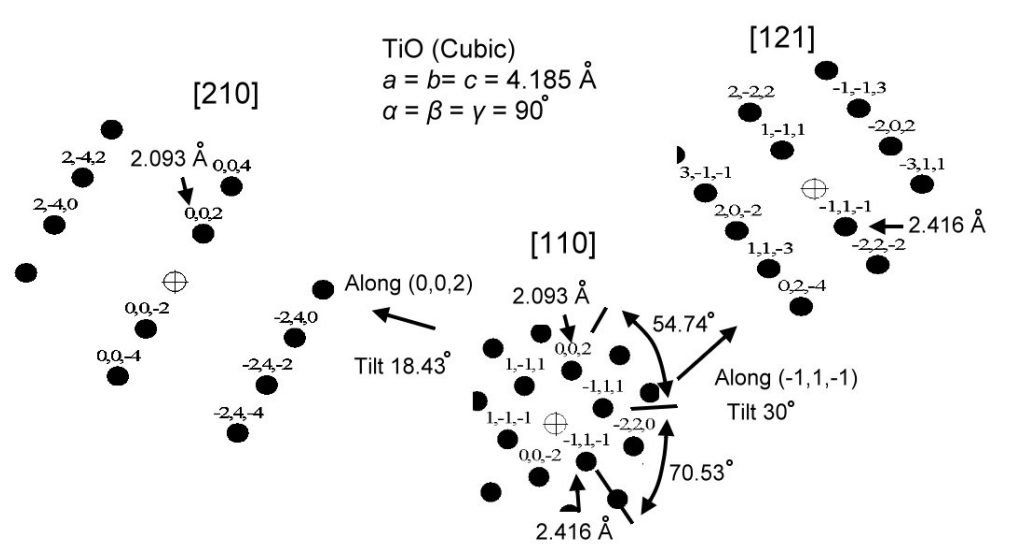

Figure 7. Simulated SADPs of TiO along the [210], [110], and [121] zone axes.

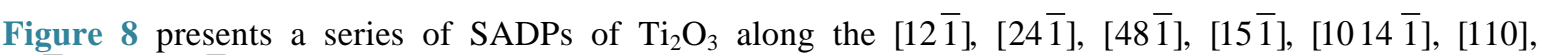
$\left[\begin{array}{lll}10 & 8 & \overline{1}\end{array}\right]$, and [75 $]$ zone axes; they match the experimental SADPs in Figure 4. First, we simulated the SADPs with the low-order index zone axes of [110] and [12 $\overline{1}$ ] of $\mathrm{Ti}_{2} \mathrm{O}_{3}$ and compared them with the experimental SADPs with the low-order zone axis, as in the zone axes (2), (4), and (8) in Figure 4. We find that the simulated SADP with the [110] zone axis matches the experimental SADP with zone axis (4) in Figure 4. For instance, in the simulated SADP with the zone axis of [110] in Figure 8, the $d$-spacings of $(1 \overline{1} 2)$ and $(\overline{114})$ are 3.734 


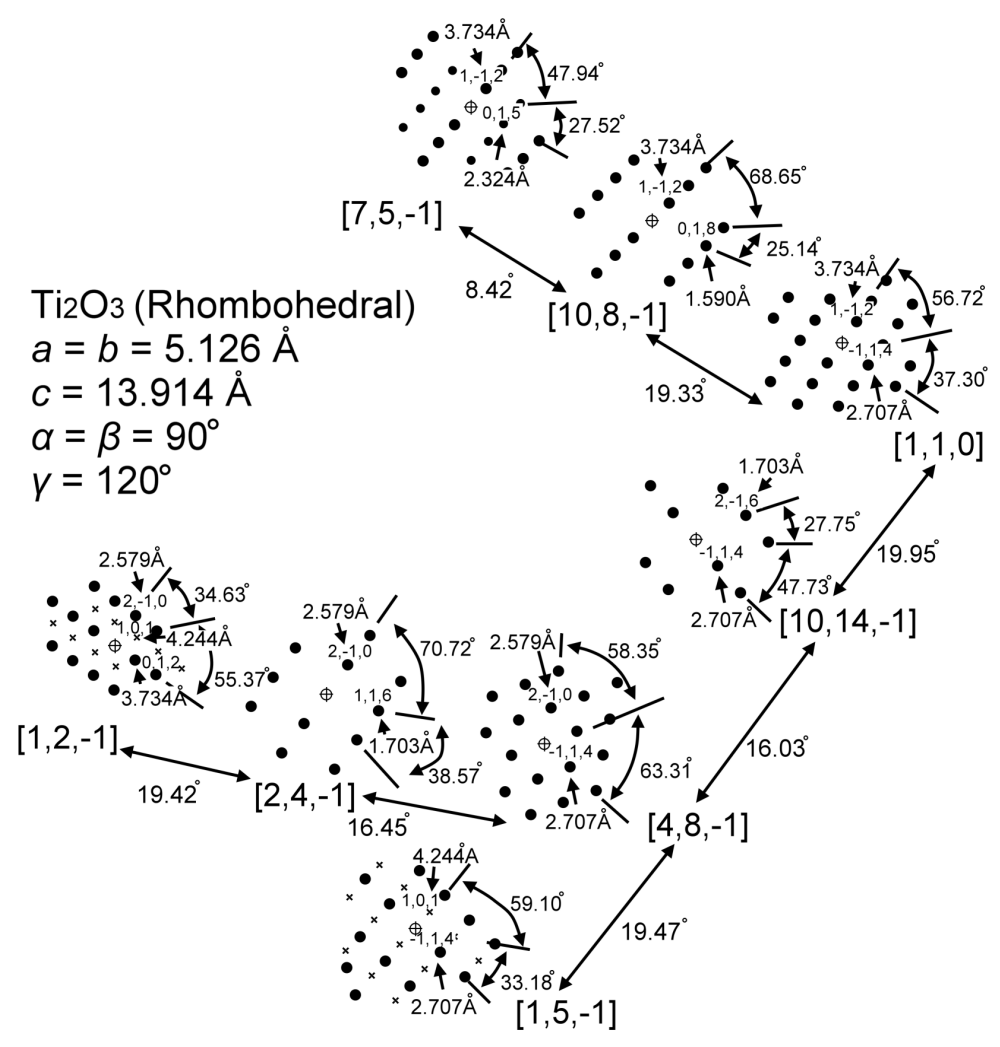

Figure 8. Simulated SADPs of $\mathrm{Ti}_{2} \mathrm{O}_{3}$ along the $[12 \overline{1}],[24 \overline{1}],[48 \overline{1}]$, $[15 \overline{1}],[1014 \overline{1}],[110],[108 \overline{1}]$, and [75 $\overline{1}]$ zone axes.

and $2.707 \AA$, respectively; for the experimental SADP of zone (4) in Figure 4, the $d$-spacings of then corresponding diffracted spots are 3.81 and $2.73 \AA$, respectively. Moreover, the angle between (1 12$)$ and (1114) of the simulated SADP of the [110] zone axis in Figure 8 is $94.02^{\circ}\left(56.72^{\circ}+37.30^{\circ}\right)$; for the experimental SADP with the zone axis (4) in Figure 4, the angle between the two nearest spots is $94.0^{\circ}\left(57.2^{\circ}+37.8^{\circ}\right)$. Next, we simulated all the SADPs of $\mathrm{Ti}_{2} \mathrm{O}_{3}$ and compared them with the experimental SADPs of the unknown $\mathrm{Ti}_{x} \mathrm{O}_{y}$ phase in Figure 4. We found that the simulated SADP of the [110] zone axis in Figure 8 was consistent with the experimental SADP with zone axis (4) in Figure 4. The next simulated SADP should be tilted along with the (114) plane of the SADP with zone [110] by approximately $19.95^{\circ}$ to get the simulated SADP with zone axis of $[1014 \overline{1}]$, tilted continuously by approximately $16.03^{\circ}$ to get the simulated SADP with the zone axis of [48 $\overline{1}$, and then tilted along the $(2 \overline{1} 0)$ plane of the simulated SADP with the zone axis of [481] to get the simulated SADP of the $[24 \overline{1}]$ and $[12 \overline{1}]$ zone axes. The tilting direction from a simulated SADP to the next simulated SADP should accord to which Kikuchi line of the SADP is tilted with respect to another SADP when recording a series of experimental SADPs through TEM observations. Thus, it is important to record the position and Kikuchi line of each experimental SADP and which Kikuchi line of the SADP was tilted (or which diffracted spot was excited) with respect to another SADP. In addition, while matching an SADP, the structure factor rules might eliminate certain diffraction spots in the SADP. For example, the diffracted spot (101), marked as " $\times$ ", disappeared in the simulated SADP with zone axes of $[12 \overline{1}]$ and $[15 \overline{1}]$. Following the matching procedure, all of the simulated SADPs of $\mathrm{Ti}_{2} \mathrm{O}_{3}$ will match with the experimental SADPs of the unknown $\mathrm{Ti}_{\mathrm{x}} \mathrm{O}_{\mathrm{y}}$ phase, including the $d$-spacings of the nearest diffracted spots, the angles between the two diffracted spots, and the tilting angles between the two zone axes. Figure 9 displays the final indexing of the SADPs of $\mathrm{Ti}_{2} \mathrm{O}_{3}$. From the EDS spectra in Figure 3(b) and the experimental and simulated SADPs in Figure 4 and Figure 9 for the unknown $\mathrm{Ti}_{\mathrm{x}} \mathrm{O}_{\mathrm{y}}$ phase, we conclude that the unknown $\mathrm{Ti}_{\mathrm{x}} \mathrm{O}_{\mathrm{y}}$ phase at the interface between the 96.4Au-3Ni-0.6Ti interlayer and YSZ of a steel/96.4Au-3Ni-0.6Ti interlayer/YSZ joint was $\mathrm{Ti}_{2} \mathrm{O}_{3}$. The mechanism of formation of $\mathrm{Ti}_{2} \mathrm{O}_{3}$ has been discussed previously [6]. 


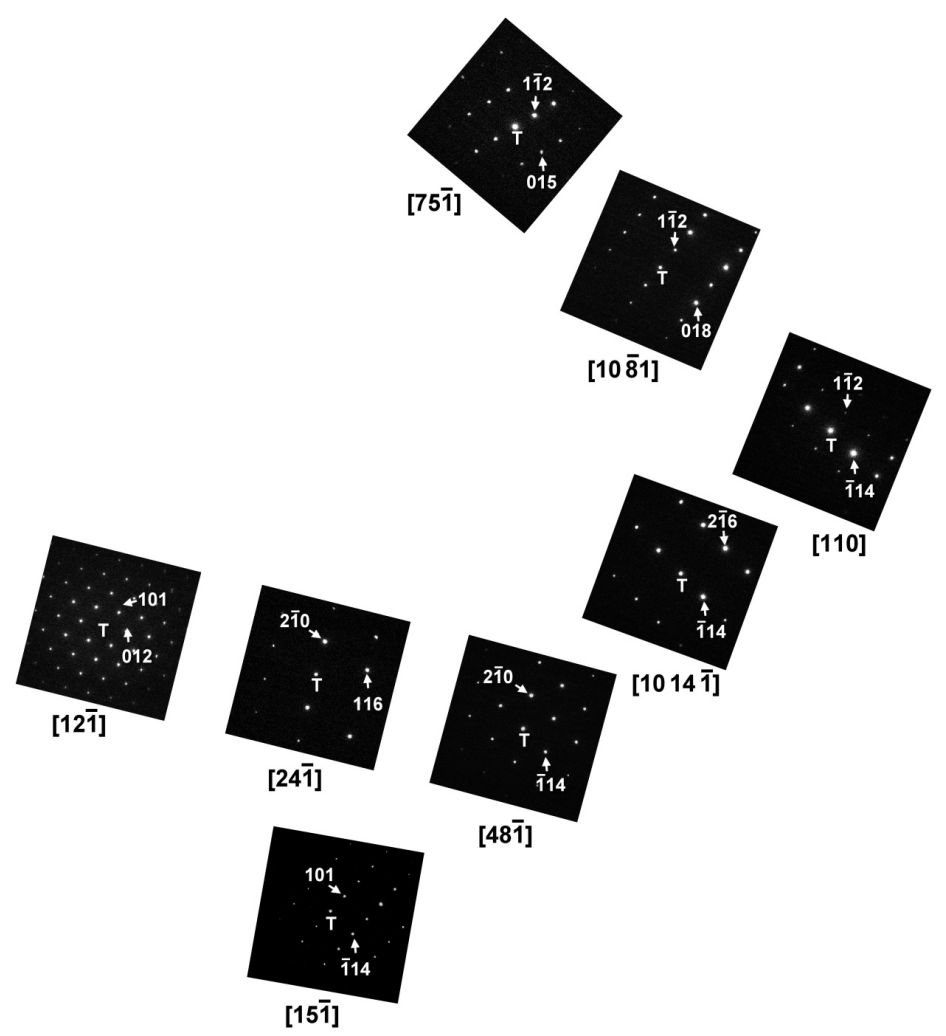

Figure 9. Series of SADPs of $\mathrm{Ti}_{2} \mathrm{O}_{3}$.
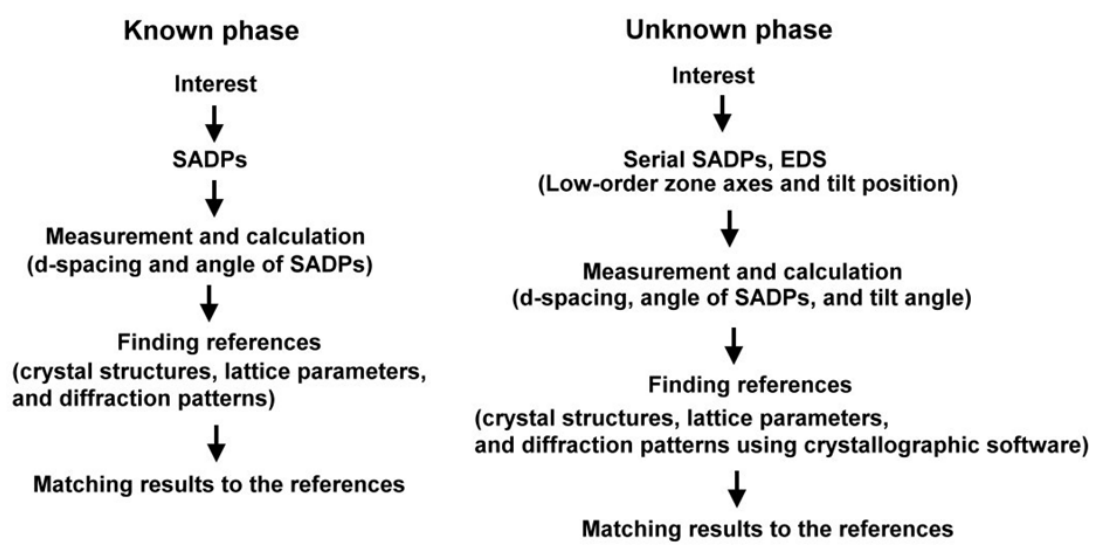

Figure 10. Procedure for using TEM to identify known and unknown phases.

Figure 10 outlines the procedure for TEM-based phase identification of known and unknown phases. For the known phase, it is necessary to capture an SADP having a low-order zone axis through TEM observation, if possible. Measurement and calculation of the $d$-spacing of the nearest diffracted spots and angle between the two zone axes is the next step. The $d$-spacing of the diffracted spots should be measured on-site in the digital film; alternatively, the camera length and electron voltage for the developed film should be recorded to calculate the $d$-spacing of the diffracted spots using the equation $R d=\lambda L$. Next, the crystallographic information of a known phase should be searched-namely, the lattice parameters, the space group, and the interplanar spacing of the $(h k l)$ plane- and referenced to the SADPs from a textbook or any crystallographic software. The final step is to match the experimental SADP with that of the reference. For an unknown phase, the EDS spectrum and a series of SADPs are required from TEM observations. The Kikuchi line and $(X, Y)$ coordinates of each SADP should be recorded on-site. The $d$-spacing of the nearest diffracted spots and the tilting angle between 
two zones should be similar to those of the known phase. Next, crystallographic information (from the crystallography open database, the ICSD, or from the internet) should be listed for all possible unknown phases. Using crystallographic software makes it easy to obtain reference SADPs for matching with possible unknown phases. The trial-and-error method can be used to match the simulated SADPs of all possible unknown phases with the experimental SADPs. The $d$-spacings of diffracted spots, the angles between two diffracted spots, and the angles between two zone axes for all simulated and experimental SADPs should be consistent.

\section{Conclusion}

We have described methods for the phase identification of a known phase, $\mathrm{Si}$, and an unknown phase, the $\mathrm{Ti}_{\mathrm{x}} \mathrm{O}_{\mathrm{y}}$ phase at the $96.4 \mathrm{Au}-3 \mathrm{Ni}-0.6 \mathrm{Ti}$ interlayer/YSZ interface in a steel/96.4Au-3Ni-0.6Ti interlayer/YSZ joint, using a series of SADPs and EDS determined through TEM. For the known phase, it was necessary to record SADPs with low-order zone axes and search for its references. For the unknown phase, we recorded a series of SADP and EDS spectra through TEM observations; in addition, we recorded the Kikuchi map and the $(X, Y)$ coordinates of a series of SADPs and low-order zone axes of SADP. We then used crystallographic software to simulate the reference SADPs of the unknown phases, based on the ICSD, to assist the indexing of the experimental SADPs.

\section{Acknowledgements}

I thank Dr. Mrityunjay Singh and Professor Rajiv Asthana for providing the sample of steel/96.4Au-3Ni-0.6Ti interlayer/YSZ joint. Research supported by National Science Council of Taiwan under Contract No. NSC 102-2221-E-492-005.

\section{References}

[1] Edington, J.W. (1974) Monographs in Practical Electron Microscopy in Materials Science. N. V. Philips, England.

[2] Williams, D.B. and Carter, C.B. (1996) Transmission Electron Microscopy. Plenum Press, New York, 267-287. http://dx.doi.org/10.1007/978-1-4757-2519-3

[3] Fultz, B. and Howe, J. (2000) Transmission Electron Microscopy and Diffractometry of Materials. Springer, Berlin, 276-284.

[4] Thomas, G. and Goringe, M.J. (1979) Transmission Electron Microscopy of Materials. John Wiley \& Sons, New York.

[5] Loretto, M.H. (1984) Electron Beam Analysis of Materials. Chapman \& Hall, London. http://dx.doi.org/10.1007/978-94-009-5540-0

[6] Lin, K.-L., Singh, M. and Asthana, R. (2014) Characterization of Yttria-Stabilized-Zirconia/Stainless Steel Joint Interfaces with Gold-Based Interlayers for Solid Oxide Fuel Cell Applications. Journal of the European Ceramic Society, 34, 355-372. http://dx.doi.org/10.1016/j.jeurceramsoc.2013.08.036 
Scientific Research Publishing (SCIRP) is one of the largest Open Access journal publishers. It is currently publishing more than 200 open access, online, peer-reviewed journals covering a wide range of academic disciplines. SCIRP serves the worldwide academic communities and contributes to the progress and application of science with its publication.

Other selected journals from SCIRP are listed as below. Submit your manuscript to us via either submit@scirp.org or Online Submission Portal.
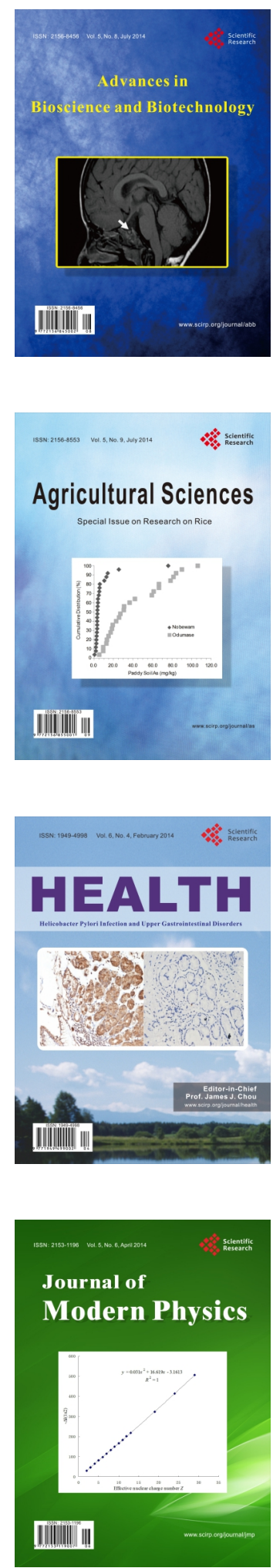
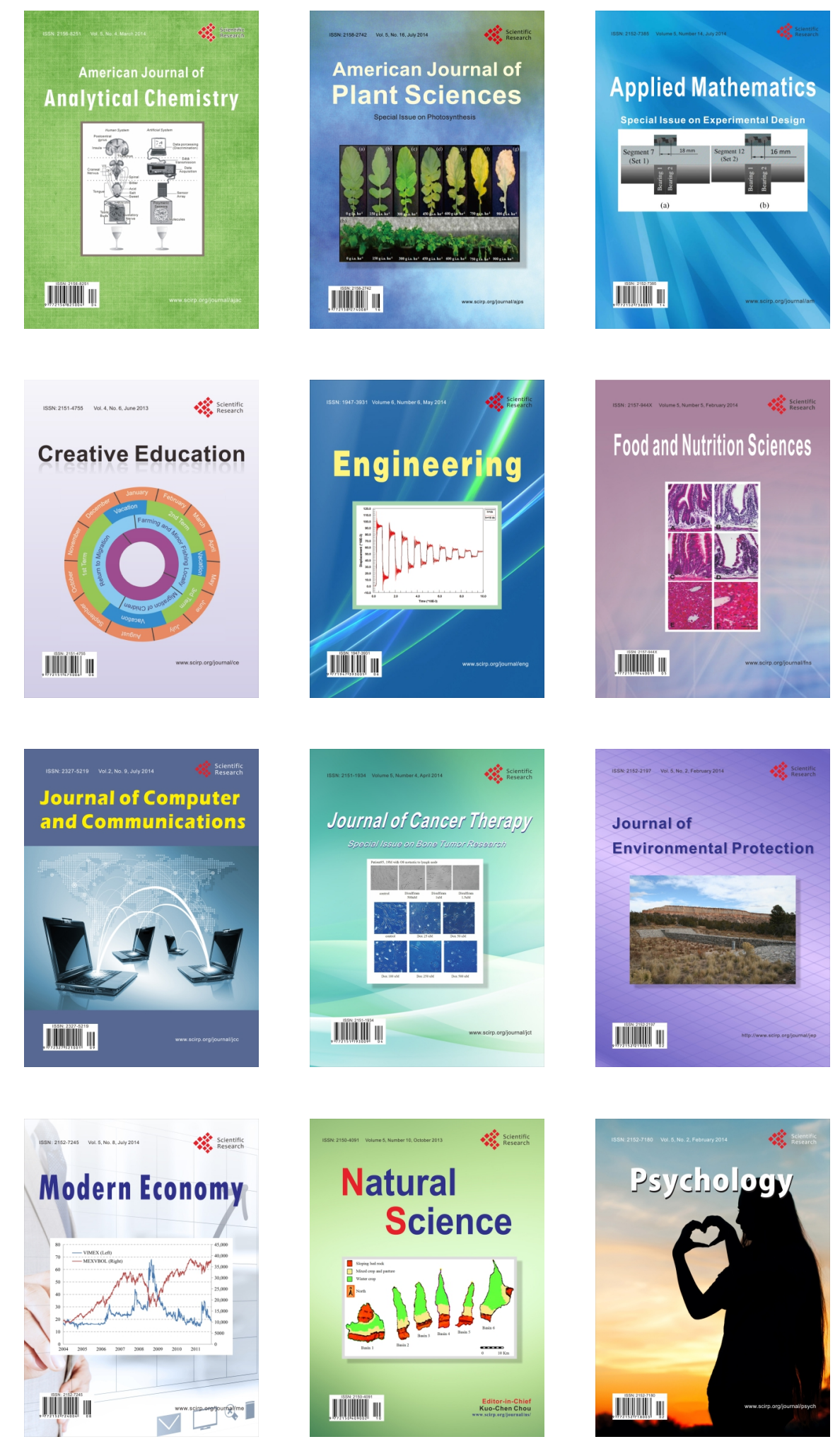